\title{
ARTICLE
}

Clinical Study

\section{Phase 1/2a trial of intravenous BAL101553, a novel controller of the spindle assembly checkpoint, in advanced solid tumours}

\author{
Rebecca Kristeleit ${ }^{1,2}$, Jeffry Evans ${ }^{3,4}$, L. Rhoda Molife ${ }^{5}$, Nina Tunariu ${ }^{5}$, Heather Shaw ${ }^{2}$, Sarah Slater ${ }^{4}$, Noor R. Md Haris ${ }^{6}$, \\ Nicholas F. Brown ${ }^{2}$, Martin D. Forster ${ }^{2}$, Nikolaos Diamantis ${ }^{5}$, Robert Rulach ${ }^{4}$, Alastair Greystoke ${ }^{6,7}$, Uzma Asghar $^{2}$, Mihaela Rata $^{8}$, \\ Stephanie Anderson ${ }^{9}$, Felix Bachmann ${ }^{9}$, Alison Hannah ${ }^{10}$, Thomas Kaindl ${ }^{9}$, Heidi A. Lane ${ }^{9}$, Patrice J. Larger ${ }^{9}$, Anne Schmitt-Hoffmann ${ }^{9}$, \\ Marc Engelhardt ${ }^{9}$, Alexandar Tzankov ${ }^{11}$, Ruth Plummer ${ }^{6,7}$ and Juanita Lopez ${ }^{5}$
}

BACKGROUND: BAL101553 (lisavanbulin), the lysine prodrug of BAL27862 (avanbulin), exhibits broad anti-proliferative activity in human cancer models refractory to clinically relevant microtubule-targeting agents.

METHODS: This two-part, open-label, phase 1/2a study aimed to determine the maximum tolerated dose (MTD) and dose-limiting toxicities (DLTs) of 2-h infusion of BAL101553 in adults with advanced or recurrent solid tumours. The MTD was determined using a modified accelerated titration design in phase I. Patients received BAL101553 at the MTD and at lower doses in the phase 2a expansion to characterise safety and efficacy and to determine the recommended phase 2 dose (RP2D).

RESULTS: Seventy-three patients received BAL101553 at doses of $15-80 \mathrm{mg} / \mathrm{m}^{2}$ (phase 1, $n=24$; phase 2a, $n=49$ ). The MTD was $60 \mathrm{mg} / \mathrm{m}^{2}$; DLTs observed at doses $\geq 60 \mathrm{mg} / \mathrm{m}^{2}$ were reversible Grade 2-3 gait disturbance with Grade 2 peripheral sensory neuropathy. In phase $2 \mathrm{a}$, asymptomatic myocardial injury was observed at doses $\geq 45 \mathrm{mg} / \mathrm{m}^{2}$. The RP2D for 2 -h intravenous infusion was $30 \mathrm{mg} / \mathrm{m}^{2}$. The overall disease control rate was $26.3 \%$ in the efficacy population.

CONCLUSIONS: The RP2D for 2-h infusion of BAL101553 was well tolerated. Dose-limiting neurological and myocardial side effects were consistent with the agent's vascular-disrupting properties.

CLINICAL TRIAL REGISTRATION: EudraCT: 2010-024237-23.

British Journal of Cancer (2020) 123:1360-1369; https://doi.org/10.1038/s41416-020-1010-8

\section{BACKGROUND}

Microtubules are present in both interphase- and dividing cells and are involved in several critical cellular functions, including mitosis, intracellular trafficking, cell signalling, migration, secretion, and angiogenesis. ${ }^{1-3}$ Microtubule-targeting agents (MTAs) can disrupt microtubule and mitotic spindle function, with subsequent anti-tumour and vascular-disrupting effects. Conventional MTAs fall into two main groups: microtubule-destabilising agents (including Vinca alkaloids, halichondrins, and combretastatins) that destabilise or depolymerise microtubules, and microtubule-stabilising agents (including taxanes and epothilones) that polymerise microtubules. ${ }^{3,4}$ At the cellular level, both groups of agents suppress spindle-microtubule dynamics, causing mitotic arrest, and trigger cell death through apoptosis. ${ }^{3}$

Microtubule-targeting agents are among the most active cytotoxic anti-cancer drugs currently in use. However, despite a high initial sensitivity of many malignancies to MTAs, resistance invariably arises through several mechanisms that are postulated to include drug efflux pump (P-glycoprotein) overexpression, aberrant expression of tubulin isotypes and BRCA1, and deregulated cell survival pathways. ${ }^{3,5-7}$ There is an urgent clinical need for novel treatment options for patients with advanced solid tumours, who are refractory to conventional MTAs.

BAL101553 (lisavanbulin) is a water-soluble lysine prodrug of the active furazano-benzimidazole derivative BAL27862 (avanbulin), a novel, synthetic molecule that shows promising anti-tumour activity. ${ }^{8,9}$ Non-clinical studies have revealed that BAL27862 acts by destabilising microtubules through a unique mechanism of action distinct from that of other MTAs, arresting tumour cell proliferation in the G2/M phase of the cell cycle and inducing apoptosis. ${ }^{9,10}$ BAL27862 shares the intradimer interface, a tubulin binding site, with colchicine and disrupts microtubule organisation thereby inducing the formation of the 'spindle assembly checkpoint. ${ }^{\prime 9,10}$

BAL101553 has demonstrated broad anti-tumour activity across a panel of cell lines and xenograft models. ${ }^{11-20}$ Importantly, BAL101553 is active in human cancer models that are refractory to standard of care therapeutics, including clinically relevant MTAs, due to P-glycoprotein overexpression and non-P-glycoproteinrelated mechanisms. ${ }^{14,15,20}$ Hence, the activity of BAL101553 does

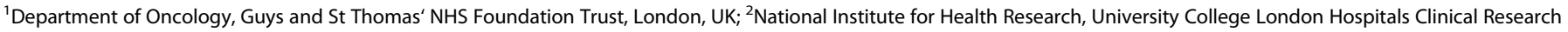

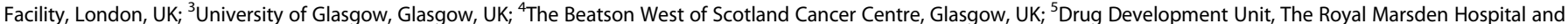

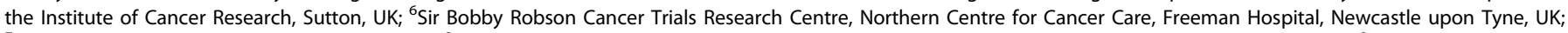

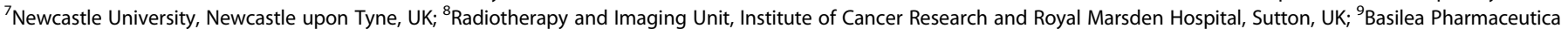
International Ltd, Basel, Switzerland; ${ }^{10}$ Oncology Clinical Trial Consulting, Sebastopol, CA, USA and ${ }^{11}$ Institute of Medical Genetics and Pathology, Basel, Switzerland Correspondence: Rebecca Kristeleit (rebecca.kristeleit@gstt.nhs.uk)

Received: 28 October 2019 Revised: 29 June 2020 Accepted: 16 July 2020

Published online: 3 August 2020 
not appear to be affected by factors that confer resistance to conventional MTAs. BAL101553 also targets the tumour microenvironment ${ }^{14}$ causing tumour vascular disruption, ${ }^{21}$ which has also been described for other MTAs. BAL101553 can be administered intravenously or orally.

Here, we report results from the first-in-human dose-escalation study of BAL101553 monotherapy in patients with advanced cancer.

\section{METHODS}

\section{Study design}

This was a two-part, open-label, phase $1 / 2$ a study of single-agent BAL101553 in patients with advanced solid tumours. The study was conducted in accordance with the Declaration of Helsinki and Good Clinical Practice. Institutional Review Boards at the four study sites in the UK approved the study and all participants provided written informed consent.

The primary objectives were to determine the maximum tolerated dose (MTD) and characterise the dose-limiting toxicities (DLTs) of single-agent BAL101553 administered intravenously over $2 \mathrm{~h}$ on days 1,8 , and 15 of a 28-day treatment cycle. Secondary objectives comprised evaluation of the safety and tolerability of BAL101553, the pharmacokinetics (PK) of BAL101553 and BAL27862, and the anti-tumour activity of BAL101553. Exploratory objectives were to characterise the pharmacodynamic effects of BAL101553/BAL27862, including functional vascular imaging, and to explore the potential utility of biomarkers in blood and/or tumour tissue for patient stratification.

The MTD of BAL101553 was determined by dose-escalation in the phase 1 part of the study. The phase $2 a$ expansion part was originally intended to characterise the safety, tolerability, and efficacy of BAL101553 at the MTD level determined in phase 1. Based on emergent data during the study, the design of the phase 2a part was modified in several amendments to investigate whether a lower dose level was clinically preferable to dosing at the MTD. The design was changed from a fixed-dose treatment of 20 patients at the MTD to an open-label randomised (1:1) design that randomised 40 patients to receive either the MTD or $50 \%$ of the MTD. Randomisation of patients during this period of the study is described in Supplementary Text 1. In subsequent amendments the tested doses were further reduced and ultimately all remaining patients were treated at the 50\% MTD dose level.

\section{Patients}

Eligible patients were aged 18 years or older with advanced or recurrent solid tumours who had failed standard therapy, or for whom no standard therapy was available. Patients who had received previous treatment with taxanes or other MTAs were permitted. In the phase 2a part of the study, inclusion was limited to patients with colorectal, gastric, or gastro-oesophageal junction, non-small-cell lung, ovarian (including primary peritoneal), pancreatic (including ampullary), or triple-negative breast cancers to limit variability. These six cancer types were selected based on clinical and/or pharmacodynamic efficacy signals in the phase 1 part of the study, and biomarker studies of human tumour tissue libraries suggested that these cancers typically express biomarkers considered relevant for the anti-tumour efficacy of BAL101553, i.e., microtubule- and spindle assembly checkpoint-related markers (such as BubR1).

BuBR1 staining was performed in available tissues from patients participating in this study. However, these were exploratory studies and not performed as a selection biomarker prior to study entry (data not shown).

All patients had measurable disease according to RECIST (Response Evaluation Criteria In Solid Tumours) criteria v1.1 documented within 35 days prior to starting study drug: an
Eastern Cooperative Oncology Group (ECOG) performance status $\leq 1$; adequate organ and marrow function; and a life expectancy of $\geq 12$ weeks.

Patients with peripheral neuropathy Grade $\geq 2$ (National Cancer Institute Common Terminology Criteria for Adverse Events [NClCTCAE] v4.03); systolic blood pressure $\geq 140 \mathrm{mmHg}$ and/or diastolic BP $\geq 90 \mathrm{mmHg}$; significant cardiac or cerebrovascular disease; those treated with a calcium channel blocker, or who required a combination of more than two antihypertensive medications to control blood pressure and patients requiring vitamin $\mathrm{K}$ antagonists were excluded from study entry. Full inclusion/exclusion criteria are listed in Supplementary Text 2.

\section{Study treatment}

BAL101553 was administered intravenously over $2 \mathrm{~h}$ on days 1, 8, and 15 of a 28-day cycle. Intravenous dosing was used as the oral formulation of BAL101553 was not available at the time of this study. Dosing on days 1, 8, and 15 over a 28-day cycle was chosen based on the expected gastrointestinal and haematological toxicity (observed in 3-week Good Laboratory Practice toxicity studies in rats and dogs with weekly intravenous administration), with the goal to have a 14-day drug-free interval to recover from such toxicity. Treatment was continued until disease progression, unacceptable toxicity or until investigator/patient decision to withdraw.

Intra-patient dose escalation to a higher dose already considered safe was allowed in the phase 1 part of the study for patients who had completed two or more cycles of BAL101553 without any Grade $\geq 2$ drug-related adverse events (AEs). Dose interruptions were allowed for a DLT until recovery to CTCAE Grade $\leq 1$ or baseline; subsequent doses of BAL101553 were reduced by one dose level. Dose interruptions and reductions were also allowed for non-DLT events. For cycle 1, if a patient did not meet the requirements for being dosed on either day 8 or day 15, the physician could delay treatment for up to 5 days. BAL101553 was administered if the patient met re-treatment criteria within these 5 days. Cycle 1 was regarded as complete if all three doses of BAL101553 were administered within 28 days, with recovery of any toxicity to permit initiation of cycle 2 with a maximum delay of 14 days.

Dose escalation. The starting weekly dose of BAL101553 $(15 \mathrm{mg} /$ $\mathrm{m}^{2}$ ) was based on Good Laboratory Practice toxicology studies in dogs and was approximately $1 / 6$ th of the highest non-severely toxic dose observed in these studies. This corresponded to a dose of approximately $27.5 \mathrm{mg}$ of BAL101553 for a $1.73 \mathrm{~m}^{2}, 70-\mathrm{kg}$ patient.

Full details of the dosing criteria are presented in Supplementary Table 1. Dose escalation in the Phase 1 part of the study was performed using a modified accelerated $3+3$ titration design. Patients were enrolled in sequential dose cohorts comprising one to six patients; cohorts were expanded if a patient experienced a BAL101553 treatment-related DLT during cycle 1. DLTs were generally defined as Grade $\geq 4$ haematological AEs or Grade $\geq 3$ non-haematological AEs (full DLT criteria are defined in Supplementary Text 3).

Dose cohort escalation decisions incorporated clinical review of all relevant available data from contemporaneous and previous dose cohorts. The maximum administered dose was defined as the dose level at which a DLT was observed during treatment cycle 1 in $\geq 33 \%$ of evaluable patients. The MTD was defined as the highest dose level below the maximum administered dose with an acceptable tolerability profile. The recommended phase 2 dose (RP2D) was determined based on all available safety, PK, pharmacodynamic, and efficacy data.

Study assessments

Safety. Safety was assessed throughout the study. Assessments included recording of all AEs (according to NCI-CTCAE v4.03) and 
1362

serious AEs (SAEs), laboratory parameters, echocardiography, vital signs, ECOG performance status, physical examination, radiology assessments, and evaluation of concomitant medications.

Pharmacokinetics. Blood samples for assessment of BAL101553 and BAL27862 pharmacokinetics were taken from patients in phase 1 of the study on day 1 of cycles 1 and 2 (pre-dose, and at 1 , 2 [immediately prior to end of infusion], 2.5, 3, 4, 6, 8, 24, and $48 \mathrm{~h}$ after the start of the 2-h infusion). In the phase 2a part of the study, samples were taken on day 1 of cycle 1 (pre-dose, 2 [immediately prior to end of infusion], 4, 6, 10, and $24 \mathrm{~h}$ after the start of the 2-h infusion). Additional samples were taken immediately prior to the end of the 2 -h infusion on days 8 and 15 of cycle 1 (and cycle 2 in phase 1), at the occurrence of a DLT, and from patients undergoing intra-patient dose escalation or dose reduction. Urine samples were taken on day 1 of cycles 1 and 2 from patients in the phase 1 part of the study for calculation of total 24-h urinary excretion of BAL101553 and BAL27862. BAL101553 and BAL27862 were quantified in plasma and urine using liquid chromatography tandem-mass spectrometry with a lower limit of quantification of $1 \mathrm{ng} / \mathrm{mL}$.

Pharmacodynamics. Blood samples obtained on days 1, 15, and 22 of cycle 1 , and day 22 of cycle 2 were evaluated for circulating tumour cell, circulating endothelial cell, and circulating endothelial progenitor cell counts. Tumour biopsies (where possible) were to be taken at screening, on day 22 of cycles 1, 2, and one subsequent cycle, and at disease progression. Tumour biopsies were analysed by immunohistochemistry for exploratory patient biomarkers. In phase 2a, serial dynamic contrast-enhanced magnetic resonance imaging (MRI) and diffusion-weighted MRI were conducted in suitable patients to assess the effect of BAL101553 on tumour vascularity and cellularity. Imaging was conducted at two pre-treatment visits (2-3 days apart) and three post-treatment visits (days 1, 2, and 8 of cycle 1 ).

Efficacy. Tumour response was assessed using computed tomography scans according to RECIST v1.1 every two cycles ( 8 weeks) in patients with measurable disease.

Statistical analyses

Study populations. The modified accelerated $3+3$ design and the exploratory nature of the phase $2 \mathrm{a}$ expansion portion did not require sample size calculation justification.

Patients eligible for determining the MTD had to have received all three doses of BAL101553 during cycle 1 (or at least one dose if the patient experienced a DLT) and been followed for $\geq 28$ days after the first dose for safety (MTD population). Safety was evaluated in all recruited patients (safety population). Pharmacokinetics were evaluated in all patients who received at least one partial or complete dose of BAL101553 and had at least one postbaseline PK assessment (PK population). Anti-tumour activity was evaluated in all patients who received all three doses of BAL101553 in cycle 1 and who either underwent at least one on-study tumour assessment or a radiological assessment by RECIST guidelines at or after the end of cycle 2, or who showed clinical and/or radiological progressive disease prior to the end of cycle 2 (efficacy population). Efficacy parameters were also analysed using the full analysis population (all patients who received at least one partial or complete dose of BAL101553 based on the intent-to-treat principle).

Statistical assessments. Demographic, baseline, safety, PK, and pharmacodynamic data were analysed by dose cohort using descriptive statistics. Pharmacokinetic parameters were calculated from plasma and urine concentration data by non-compartmental analysis using Phoenix WinNonLin 7.0. Anti-tumour activity was assessed by dose cohort summarising the objective response rate
(ORR), disease control rate (DCR), and progression-free survival (PFS) with exact 95\% confidence intervals $(\mathrm{Cl})$. The ORR was defined as complete response plus partial response (PR); the DCR was defined as complete response plus PR plus stable disease (SD) lasting two or more cycles from start of BAL101553 treatment to earliest date of clinical/objective progression. Progression-free survival was defined as the interval between the date of first infusion and the earliest date of clinical/objective progression or death. Median time to progression or death was also determined by the Kaplan-Meier method.

\section{RESULTS}

Patient demographics and disposition

Seventy-three patients were enrolled at four sites in the UK. The first patient, first visit was on 12 July 2011 and the last patient, last visit was on 6 April 2016. Baseline demographic and disease data are presented in Table 1 and by dose group in Supplementary Table 2. Overall, $53.4 \%$ of patients were male, $90.4 \%$ were white Caucasian and median age was 59 years (range 29-80). ECOG performance status at baseline was 0 for $23(31.5 \%)$ patients and 1 for $50(68.5 \%)$ patients. The most prevalent cancer types were colorectal cancer (30.1\%), non-small-cell lung cancer (12.3\%), and pancreatic/ampullary cancer (12.3\%). Thirty-four (46\%) patients had previously been treated with MTAs, of which 24 had discontinued treatment due to documented disease progression.

Phase 1

All patients were treated with at least one dose of BAL101553 at dose levels between 15 and $80 \mathrm{mg} / \mathrm{m}^{2}$. Twenty-four patients received BAL101553 at starting dose levels of $15 \mathrm{mg} / \mathrm{m}^{2}(n=1)$, $30 \mathrm{mg} / \mathrm{m}^{2}(n=3), 45 \mathrm{mg} / \mathrm{m}^{2}(n=3), 60 \mathrm{mg} / \mathrm{m}^{2}(n=10)$, and 80 $\mathrm{mg} / \mathrm{m}^{2}(n=7)$. Two of six evaluable patients treated at a starting dose of $80 \mathrm{mg} / \mathrm{m}^{2}$ experienced a DLT of reversible Grade 2-3 gait disturbance. The gait disturbance in both cases occurred with fully or partially reversible Grade 2 peripheral sensory neuropathy. The reduction in proprioception/sensation contributed to the gait disturbance. Therefore, the MTD for BAL101553 administered as a 2-h infusion on days 1,8 , and 15 of a 28-day cycle was determined to be $60 \mathrm{mg} / \mathrm{m}^{2}$ and the maximum administered dose was $80 \mathrm{mg} /$ $\mathrm{m}^{2}$. One of the six evaluable patients treated at the MTD experienced a similar toxicity (Grade 3 reduced mobility with dizziness).

Phase 2a

Following the changes in study design, patients were randomised to receive BAL101553 in the phase 2a part at either the MTD (60 $\left.\mathrm{mg} / \mathrm{m}^{2}\right)$ or $50 \%$ of the MTD $\left(30 \mathrm{mg} / \mathrm{m}^{2}\right)$. Three additional AEs meeting the criteria for a DLT were reported in the phase 2a part of the study. These events necessitated that the maximum dose investigated in phase $2 \mathrm{a}$ was reduced to $45 \mathrm{mg} / \mathrm{m}^{2}$ after two patients treated at $60 \mathrm{mg} / \mathrm{m}^{2}$ experienced myocardial injury (Grade 3 troponin elevation and electrocardiogram changes including T-wave inversions). Subsequently, a further patient treated at $45 \mathrm{mg} / \mathrm{m}^{2}$ experienced asymptomatic myocardial infarction and the dose cohort level was lowered to $30 \mathrm{mg} / \mathrm{m}^{2}$. Overall, 49 patients received BAL101553 at starting dose levels of $30 \mathrm{mg} / \mathrm{m}^{2}(n=33), 45 \mathrm{mg} / \mathrm{m}^{2}(n=5)$, and $60 \mathrm{mg} / \mathrm{m}^{2}(n=11)$ in the phase $2 \mathrm{a}$ expansion part. The RP2D for $2-\mathrm{h}$ intravenous infusion of BAL101553 was determined to be $30 \mathrm{mg} / \mathrm{m}^{2}$.

\section{Overall safety data}

The median duration of study-drug treatment was 43.0 days (range 1-1032) and two cycles (range 1-37). Four (5.5\%) patients withdrew consent: two due to DLTs, one due to the time-intensive schedule of the trial, and one for whom no reason was given. Six (8.2\%) patients died during the study or within 30 days following the last dose. One patient died during screening and was never 
Table 1. Baseline demographic and disease history data of the 73 enrolled and treated patients.

\begin{tabular}{ll}
\hline & Total $(n=73)$ \\
\hline Age (years), median (range) & $59.0(29-80)$ \\
Female/Male, $n$ (\%) & $34(46.6) / 39(53.4)$ \\
ECOG PS 0/1, $n$ (\%) & $23(31.5) / 50(68.5)$ \\
Prior treatment regimens & \\
Overall, median (range) & $3(0-8)$ \\
Chemotherapy/hormone therapy, $n$ (\%) & $72(98.6)$ \\
Prior MTAs, $n$ (\%) & $34(46.6)$ \\
Radiotherapy, $n$ (\%) & $29(39.7)$ \\
Surgery, $n$ (\%) & $52(71.2)$ \\
Most common tumour types & \\
Colorectal & $22(30.1)$ \\
NSCLC & $9(12.3)$ \\
Pancreatic/ampullary cancer & $9(12.3)$ \\
Gastro-oesophageal cancer & $8(11.0)$ \\
Ovarian/primary peritoneal cancer & $8(11.0)$ \\
TNBC & $4(5.5)$ \\
Other & \\
Tumour histology, $n$ (\%) & $13(17.8)$ \\
Adenocarcinoma & \\
Squamous cell carcinoma & \\
Other & $59(80.8)$ \\
Metastatic disease, $n$ (\%) & $3(4.1)$ \\
Histopathological grade, $n$ (\%) & $11(15.1)$ \\
Grade 1 & $66(91.7)$ \\
Grade 2 & \\
Grade 3 & 0 \\
Not assessable & $24(33.8)$ \\
Other & $19(26.8)$ \\
Missing & $16(22.5)$ \\
\hline ECOG PS Eate & $12(16.9)$ \\
\hline & 2 \\
\hline
\end{tabular}

ECOG PS Eastern Cooperative Oncology Group performance status, MTA microtubule-targeting agent, NSCLC non-small-cell lung cancer, $T N B C$ triplenegative breast cancer.

a Other comprises: two cases of oesophageal cancer and single cases of adrenocortical cancer, anal cancer, cervical cancer, cholangiocellular cancer, epitheliod mesothelioma, gastric cancer, laryngeal cancer, mesenchymal chondrosarcoma, neuroendocrine cancer, small bowel cancer, thymoma.

dosed. The other five deaths occurred within 28 days of the last study drug administration; four were due to disease progression and judged not to be drug related. One patient $\left(30 \mathrm{mg} / \mathrm{m}^{2}\right.$ BAL101553) died due to a suspected unexpected serious adverse reaction of acute abdomen, which, although primarily attributed to cancer progression (gastro-oesophageal junction carcinoma with liver metastases), was assessed as possibly related to BAL101553.

Adverse events judged to be drug-related occurred in 65 $(89.0 \%)$ patients (Table 2). The most common were nausea (43.8\%), vomiting (34.2\%), hypertension (32.9\%), fatigue $(31.5 \%)$, diarrhoea $(30.1 \%)$, peripheral neuropathy $(17.8 \%)$, and decreased appetite (17.8\%). Twenty-two (30.1\%) patients experienced 69 treatment-related and dose-dependent severe (Grade 3-4) events. Hypertension was the most common Grade 3-4 treatment-related $A E$, occurring in $18(24.7 \%)$ patients. Five out of seven $(71.4 \%)$ evaluable patients in the $80 \mathrm{mg} / \mathrm{m}^{2}$ cohort and 10 out of $21(47.6 \%)$ patients in the $60 \mathrm{mg} / \mathrm{m}^{2}$ cohort experienced Grade 3/4 hypertension. Only one (2.8\%) patient treated at the RP2D $\left(30 \mathrm{mg} / \mathrm{m}^{2}\right)$ experienced Grade 3 hypertension.

Serious AEs were reported in 36 (49.3\%) patients. Sixteen SAEs were considered possibly or probably treatment-related in 13 $(17.8 \%)$ patients. Pyrexia $(n=3)$, tumour pain $(n=3)$, hypertension $(n=2)$, and acute coronary syndrome/myocardial infarction $(n=2)$ were the only treatment-related SAEs to occur in more than one patient.

Thirteen (17.8\%) patients discontinued due to AEs including five (6.8\%) patients for whom the AE was considered to be treatment related. Only one $A E$ led to study drug discontinuation in more than one patient, intestinal obstruction which occurred in three (4.1\%) patients treated at 30,60 , and $80 \mathrm{mg} / \mathrm{m}^{2}$, none of which were judged to be related to the study drug. Overall, 22 (30.1\%) patients experienced treatment-emergent AEs that led to dose interruptions or modifications.

There was no apparent difference in the proportion of patients experiencing AEs when stratified by prior MTA exposure or not, and no significant odds ratio observed for any of the treatmentemergent AEs between the groups (data not shown).

Pharmacokinetics

Seventy-three patients were evaluable for PK on cycle 1, day 1, and 21 patients on cycle 2, day 1 . The low number of evaluable patients on cycle 2, day 1 was due to patient discontinuations during cycle 1 due to either progressive disease or adverse events. Overall, the exposure of BAL101553 and BAL27862 was dose related (Fig. 1a, b, Supplementary Fig. 1A, B) and similar between male and female patients (Supplementary Fig. 2A, B).

The prodrug BAL101553 was rapidly converted to BAL27862 in all subjects and at all doses. In the RP2D $\left(30 \mathrm{mg} / \mathrm{m}^{2}\right)$ cohort, the BAL101553 prodrug had a moderate plasma clearance (CL: geometric mean $19.3 \mathrm{~L} / \mathrm{h})$ and low volume of distribution $\left(V_{s s}\right.$ : $20.1 \mathrm{~L})$, resulting in a short half-life $(1.96 \mathrm{~h})$. The active drug BAL27862 had a low apparent clearance (CL/F: $10.1 \mathrm{~L} / \mathrm{h})$ and moderate apparent volume of distribution $\left(V_{\mathrm{z}} / \mathrm{F}: 182 \mathrm{~L}\right)$, resulting in a half-life of $12.6 \mathrm{~h}$.

Urinary excretion was not a significant route of elimination with $<0.1 \%$ of the administered dose of BAL101553 and $<1 \%$ of the equivalent dose for BAL27862 recovered in the urine (Supplementary Table 3). The PK parameters for BAL101553 and BAL27862 on day 1 , cycle 1 are shown in Table 3 and Supplementary Table 4. The curves for all cohorts and PK days are shown in Supplementary Figs. 2A, B, 3.

Pharmacokinetic/adverse reaction relationships. A dose relationship was observed for nausea/vomiting, transient arterial hypertension, peripheral sensory neuropathy, and pain at tumour site. Arterial hypertension was significantly higher in patients treated with $60-80 \mathrm{mg} / \mathrm{m}^{2}$ BAL 101553 than at $15-30 \mathrm{mg} / \mathrm{m}^{2}$ (Fig. 1c) and subsided over the course of several hours after the end of the 2-h intravenous study-drug administration. Peak blood pressure elevations occurred when the concentrations of the active drug BAL27862 were highest (during the first hours after the end of the 2-h infusion).

Pharmacodynamics

Functional MRI was conducted in eight $(11.0 \%)$ patients, six of whom had complete datasets and were evaluable (five treated at $30 \mathrm{mg} / \mathrm{m}^{2}$ and one at $45 \mathrm{mg} / \mathrm{m}^{2}$ ). There was no significant difference in cohort mean values for $K^{\text {trans }}$ (volume transfer constant; $p=0.95$ ), IAUGC $_{60}$ (the integrated area under the gadolinium curve in tissue over $60 \mathrm{~s} ; p=0.93$ ) and the Apparent Diffusion Coefficient ( $p=0.92$ ) across the five imaging time points, suggesting no observable treatment effect on dynamic contrastenhanced MRI and diffusion-weighted MRI parameters within this cohort. 


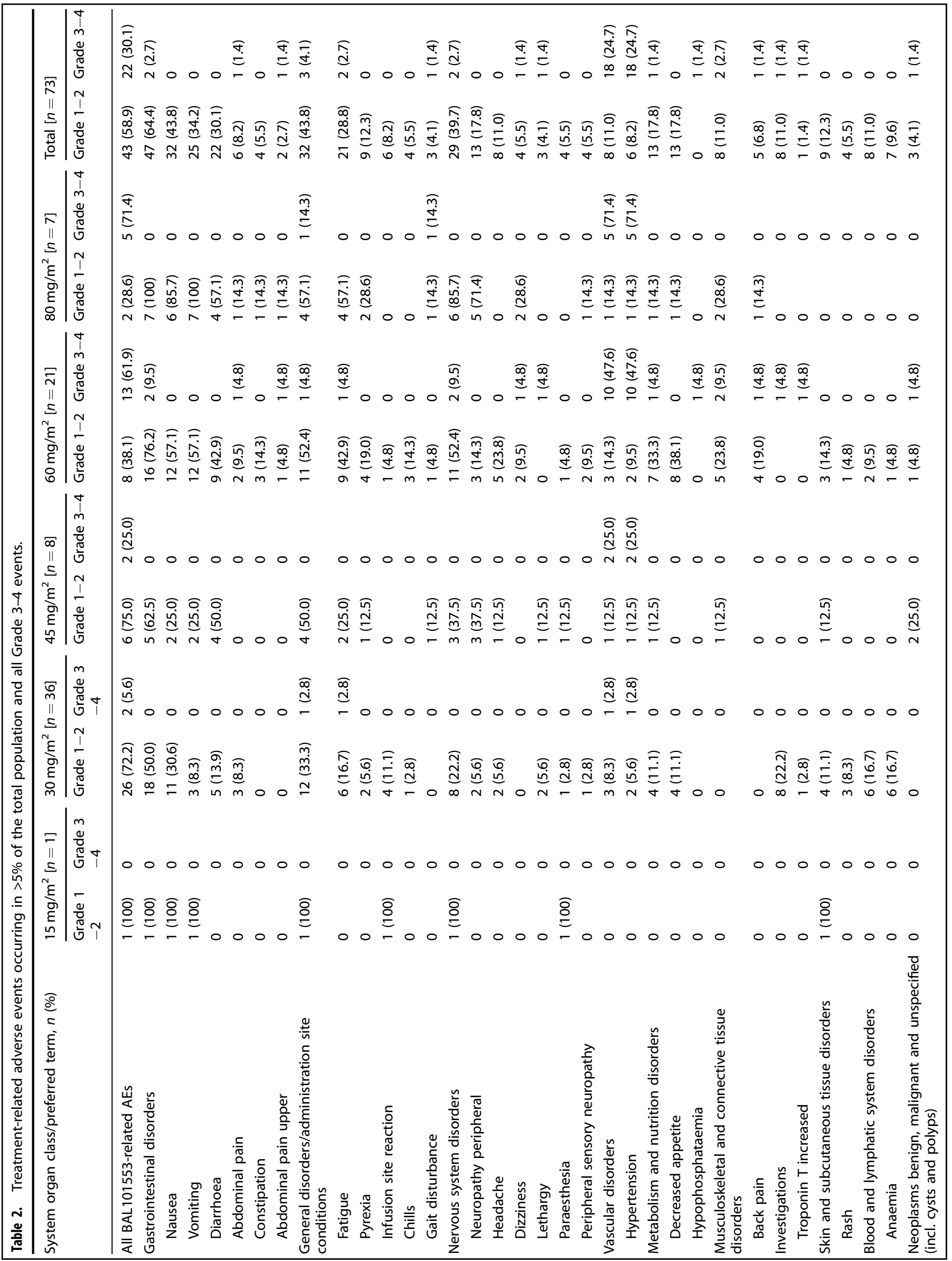


a
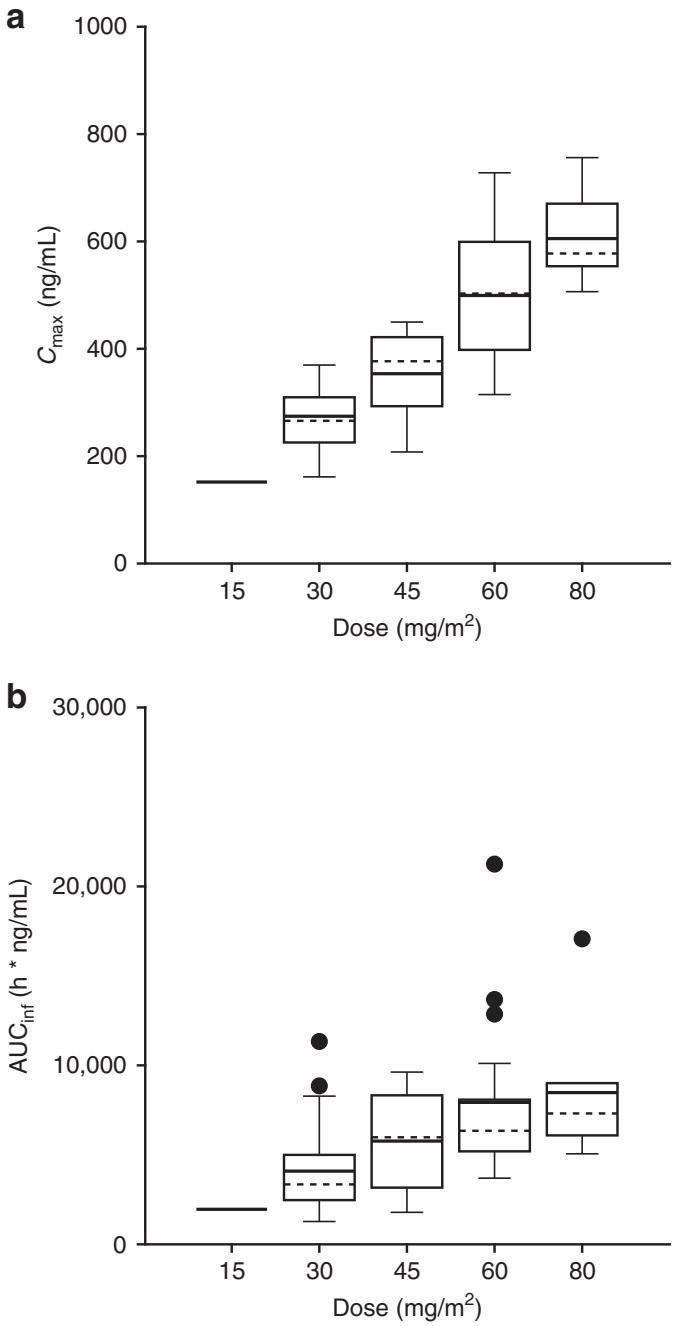

c
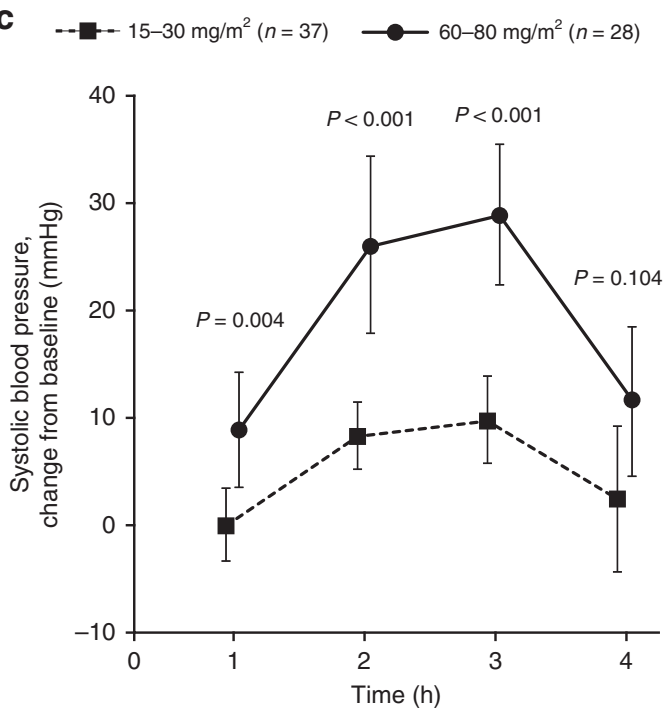

Fig. 1 Pharmacokinetic profile of BAL27862 following administration of the prodrug BAL101553. BAL27862 exposure (a $C_{\max }$ and b $\left.A \cup C_{i n f}\right)$ and c mean change from baseline in systolic blood pressure according to BAL101553 dose at cycle 1 , day $1(N=73)$. Box plots in (a, b) show median (solid horizontal line), mean (dashed horizontal line), interquartile range (box), 1.5 times the interquartile range (whiskers), and outliers (circles). Error bars in (c) indicate $95 \%$ confidence intervals. 
Table 3. Summary of BAL27862 PK parameters for cycle 1, day 1 across dose cohorts.

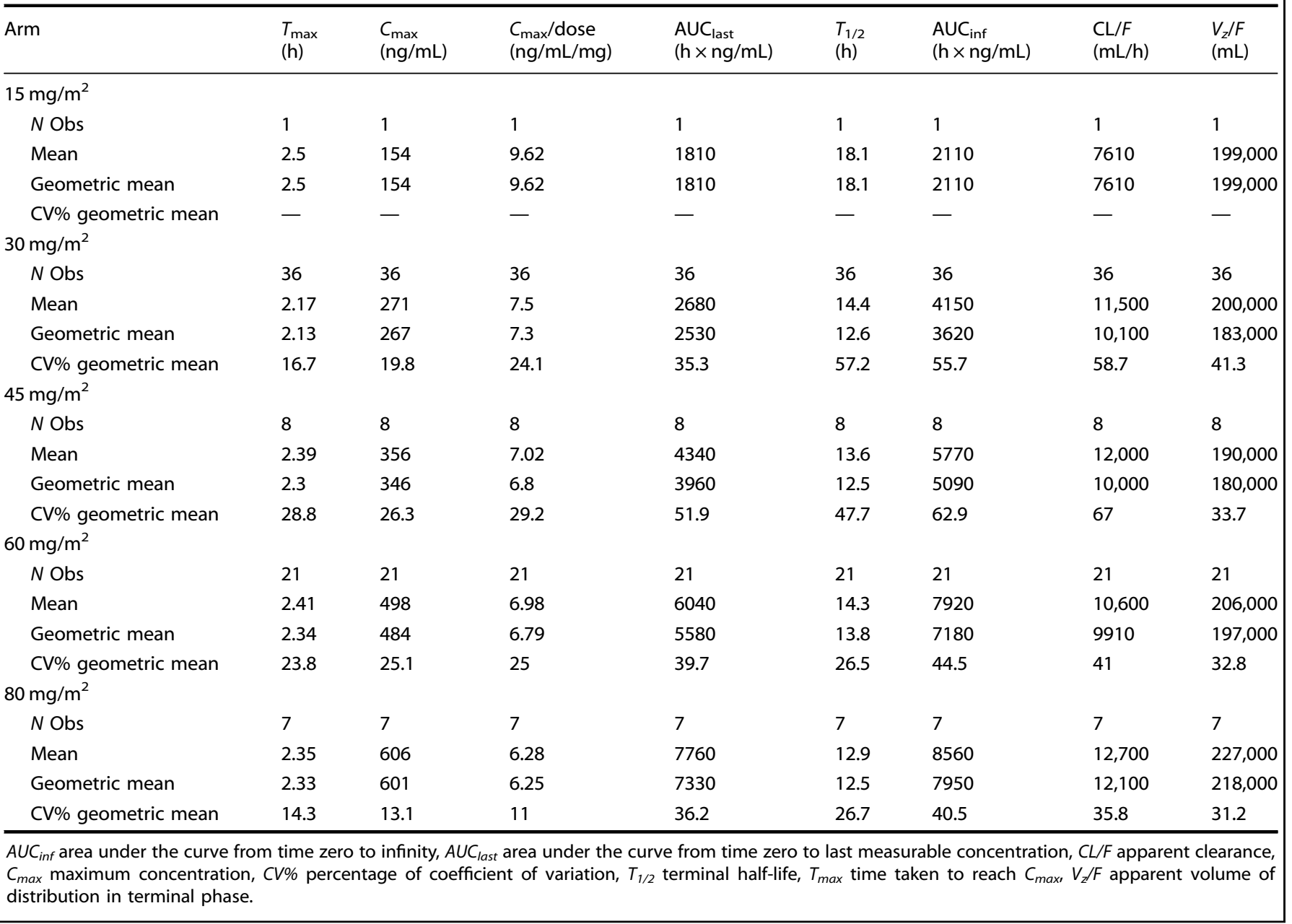

Twenty-four patients provided 1-3 pre- and post-treatment biopsies (median $=2$ ). Evaluable pairs of pre- and post-treatment biopsies were available from six patients. Reductions in tumour microvascular density (five patients, based on CD34 staining) and/ or decreases in tumour cell proliferation (two patients, based on Ki67 staining) were apparent (Supplementary Table 5 and Supplementary Figs. 4, 5). There was no clear dose effect regarding the change from baseline in enumerations of circulating tumour cells, circulating endothelial cells, or circulating endothelial progenitor cells.

\section{Efficacy}

Overall, 57 (78.1\%) patients were evaluable for efficacy. One patient with an ampullary carcinoma had a PR (starting dose level of $30 \mathrm{mg} / \mathrm{m}^{2}$ with subsequent dose escalation to $45 \mathrm{mg} / \mathrm{m}^{2}$ ). This PR lasted for over 2 years. The ORR was therefore $1.8 \%(95 \% \mathrm{Cl}$ : $0.0,9.4)$. Fourteen $(24.6 \%)$ other patients had SD lasting two or more cycles (median 15 weeks; range 7-33) as the best response (including four with SD lasting for four or more cycles), leading to an overall DCR of $26.3 \%$ (95\% Cl: $15.5,39.7 ; n=15)$. The overall median PFS was 50.0 days ( $95 \% \mathrm{Cl}: 50.0,51.0)$. The PR, SD, and progressive disease rates in patients who received BAL101553 at the recommended phase 2 dose of $30 \mathrm{mg} / \mathrm{m}^{2}(n=36)$ were $3.2 \%$, $16.1 \%$, and $80.6 \%$, respectively, equating to an ORR of $3.2 \%(95 \%$ $\mathrm{Cl}: 0.1,16.7)$ and a DCR of $19.4 \%(95 \% \mathrm{Cl}: 7.5,37.5)$ in these patients. There was no apparent relationship between tumour type and response. Overall, four of $53(7.5 \%)$ evaluable patients had reductions in the sum of longest diameter from baseline for target lesions at least once during the study. These comprised the patient with ampullary carcinoma (target lesion: mesenteric lymph node; $-73.3 \%$ change) and a patient with pancreatic cancer (two target lesions: liver, $-22.6 \%$ change) treated at $30 \mathrm{mg} / \mathrm{m}^{2}$; a patient with NSCLC (two target lesions: lung, $-1.3 \%$ change) treated at $45 \mathrm{mg} / \mathrm{m}^{2}$; and a patient with cervical cancer (target lesion: hilar lymph node, $-12.5 \%$ change) treated at $60 \mathrm{mg} / \mathrm{m}^{2}$. The overall median maximum change in the sum of longest diameter from baseline was $17.9 \%$ (range -73.3 to $171 \%$; Fig. 2). There was no clear difference in efficacy between patients with/ without prior MTA treatment. The patient with ampullary carcinoma who had a PR had not had prior MTA treatment.

\section{DISCUSSION}

This was a two-part open-label study in patients with solid tumours designed to determine the MTD and DLT of a 2-h infusion of BAL101553, the lysine prodrug of BAL27862, and to investigate the overall safety tolerability, PK profile, and anti-tumour activity. The primary objective of the study was met by establishing the MTD for BAL 101553 as initially $60 \mathrm{mg} / \mathrm{m}^{2}$ when administered intravenously over $2 \mathrm{~h}$ as single agent on days 1,8 , and 15 of a 28day treatment cycle. The RP2D was determined as $30 \mathrm{mg} / \mathrm{m}^{2}$.

The DLTs were reversible Grade $2-3$ gait disturbance at dose levels $\geq 60 \mathrm{mg} / \mathrm{m}^{2}$, occurring with Grade 2 peripheral sensory neuropathy and asymptomatic myocardial injury at dose levels $\geq 45 \mathrm{mg} / \mathrm{m}^{2}$. Most of the Grade 3 neurological events and all cardiac events occurred during or after the first infusion of study 


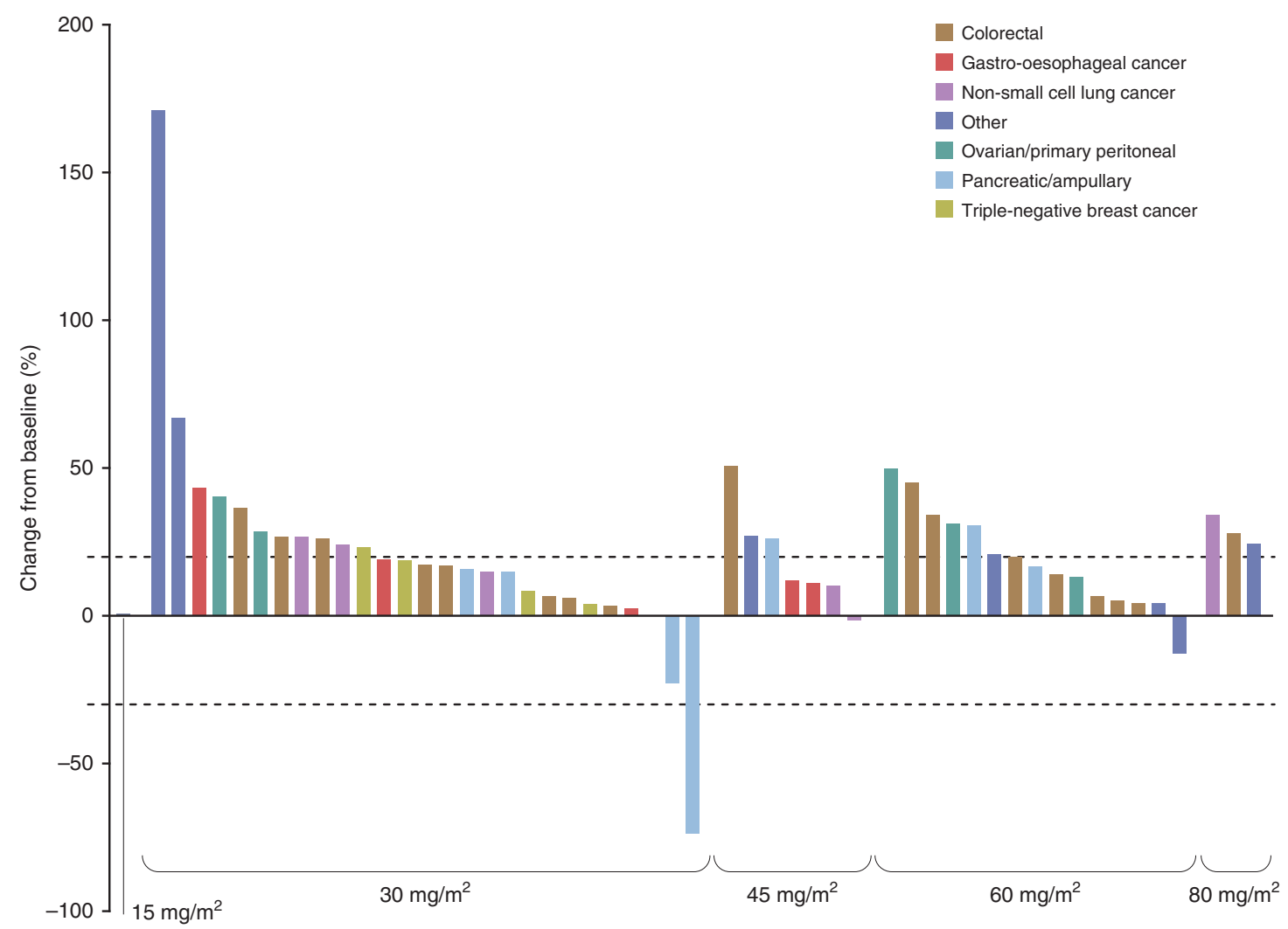

Fig. 2 Waterfall plot of best percentage change from baseline in sum of longest diameter for target lesions ( $N=53$ evaluable patients). Upper dotted line indicates the RECIST v1.1 criteria for progressive disease; lower dotted line indicates the RECIST v1.1 criteria for partial response.

drug and appeared to be related to the $C_{\max }$ of BAL101553/ BAL27862.

Peripheral neuropathy is common with MTA therapy due to their axonal microtubule disrupting activity. ${ }^{2,5,22}$ In this study, Grade 2 peripheral sensory neuropathy with reduced proprioception/sensation was observed in two patients treated with $80 \mathrm{mg} /$ $\mathrm{m}^{2}$ BAL101553 and contributed to the DLTs of Grade 2-3 gait disturbance in these patients. At the RP2D $\left(30 \mathrm{mg} / \mathrm{m}^{2}\right)$, the incidence of Grade $1 / 2$ peripheral neuropathy was relatively low $(5.6 \%)$ and reversible, and no instances of Grade 3/4 peripheral neuropathy were reported. Furthermore, the incidences of other nervous system disorders commonly associated with MTA therapy, such as headache and dizziness, ${ }^{3}$ were low following treatment with BAL101553.

Cardiovascular toxicity was not seen in standard non-clinical toxicity studies including a telemetered dog study. However, several study-design changes were required to reflect the doserelated cardiac and vascular effects observed during the study and non-clinical data which arose while the study was ongoing that indicated a dose-related vascular-disrupting effect of BAL101553 in animal models. The changes to the protocol resulted in lower doses of BAL101553 being investigated in the phase 2a part of the study. The dose in the MTD-arm was reduced to $45 \mathrm{mg} / \mathrm{m}^{2}$ and then to $30 \mathrm{mg} / \mathrm{m}^{2}$, following the observation of clinical cardiovascular toxicity in three patients. The three instances of asymptomatic myocardial injury at dose levels $\geq 45 \mathrm{mg} / \mathrm{m}^{2}$ were interpreted as microvascular myocardial damage due to the vascular-disrupting properties of BAL101553/BAL27862. The incidence and the time course of blood pressure elevations provide further evidence for a $C_{\text {max }}$-related vascular-disrupting effect of BAL101553. Rises in blood pressure predominantly occurred at dose levels $\geq 45 \mathrm{mg} / \mathrm{m}^{2}$. These elevations were transient and generally resolved within $24 \mathrm{~h}$ following the intravenous dose.
Blood pressure elevations were uncommon in patients treated at doses of $15-30 \mathrm{mg} / \mathrm{m}^{2}$. The early occurrence of troponin elevations (peak levels were observed at $8 \mathrm{~h}$ after the end of infusion) is also consistent with a $C_{\text {max }}$-related vascular effect, as is the reduction in the number of tumour microvessels in paired (pre- vs. post-dose) tumour biopsies. BAL101553 appears to be inducing significant microvascular changes, which may also explain the increasing occurrence of certain AEs such as hypertension with increasing dose.

Systemic side effects of BAL101553 that showed a clear dose relationship included nausea/vomiting, transient arterial hypertension, peripheral sensory neuropathy, gait disturbance, myocardial ischaemia/infarction, and possibly abdominal/tumour-related pain. These side effects were absent or clinically not relevant at dose levels of up to $30 \mathrm{mg} / \mathrm{m}^{2}$ but occurred with a low prevalence at the dose level of $45 \mathrm{mg} / \mathrm{m}^{2}$ and a high prevalence at the dose levels of 60 and $80 \mathrm{mg} / \mathrm{m}^{2}$. Events observed at the higher dose levels usually required clinical monitoring, additional diagnostic measures, and occasionally, dose reductions or study drug discontinuation (for peripheral neuropathy, gait disturbance, and myocardial injury) and therapeutic interventions (including antiemetic, antihypertensive treatment, analgesic treatment, or cardiac treatment equivalent to the treatment of an acute coronary syndrome). Additional systemic side effects considered characteristic of BAL101553, but which did not show a clear dose relationship, included anorexia, diarrhoea, fatigue, and pyrexia.

The incidence of gastrointestinal disturbances with BAL101553, namely nausea, vomiting and diarrhoea, and fatigue, was in keeping with those of other MTAs, particularly at doses above 30 $\mathrm{mg} / \mathrm{m}^{2}$. Myeloid toxicity, particularly neutropenia, is a common and often severe side effect observed in MTA-based combination regimens. ${ }^{2}$ No AEs of neutropaenia were reported with BAL101553 in this study. The only blood/lymphatic system disorder observed 
was Grade 1-2 anaemia, occurring at a very low incidence (9.6\%) and appearing to be unrelated to BAL101553 dose.

Overall, the RP2D of $30 \mathrm{mg} / \mathrm{m}^{2}$ determined in this study was well tolerated and showed signals of anti-tumour activity. The only Grade 3 drug-related toxicities at this level were fatigue and rare cases of transient hypertension (in patients with pre-existing hypertension). No relevant, drug-related neurological or cardiac toxicities were observed and there were only small effects on blood pressure. At this dose, with the largest number of patients ( $n=36$ on cycle 1, day 1), PK exposure showed low to moderate variability with geometric mean $\mathrm{CV} \%$ of $<20 \%$ for $C_{\max }$ and $<60 \%$ for AUC $_{\text {inf. }}$

The single PR in the patient with an ampullary carcinoma treated at $30 \mathrm{mg} / \mathrm{m}^{2}$ and escalated to $45 \mathrm{mg} / \mathrm{m}^{2}$ was long lasting. The PR response occurred at the starting dose level of $30 \mathrm{mg} / \mathrm{m}^{2}$ and this patient remained on treatment for 36 cycles before discontinuing due to the development of an allergic reaction to the study drug.

The 2-h intravenous dosing strategy is no longer being pursued; however, further investigation of BAL101553 is underway, exploring alternative dosing strategies to minimise the $C_{\text {max }}$-related vascular toxicity, including oral dosing (EudraCT 2014-003371-34, NCT02490800) and administration as a 48-h continuous infusion (NCT02895360 ${ }^{23}$ ). Encouraging data have been reported on the use of BAL101553 in combination with radiotherapy in paclitaxel and epothiloneresistant ${ }^{14}$ and orthotopic glioblastoma ${ }^{19}$ tumour models. A study of BAL101553 in combination with radiation therapy in patients with newly diagnosed glioblastoma (NCT03250299) is currently underway, and further investigations in patients with ovarian cancer and in patients with recurrent glioblastoma are planned.

In conclusion, BAL101553 is a novel spindle assembly checkpoint controller with both anti-proliferative and vasculardisrupting effects. BAL101553 given weekly as a 2-h infusion exhibited dose-limiting neurological and cardiovascular effects at dose levels $\geq 45 \mathrm{mg} / \mathrm{m}^{2}$ that appear to be related to maximum plasma concentration. The recommended phase 2 dose of $30 \mathrm{mg} /$ $\mathrm{m}^{2}$ was well tolerated and showed preliminary signals of antitumour activity.

\section{ACKNOWLEDGEMENTS}

We thank the patients and their families for their participation in this study and the staff at the study sites. The Beatson West of Scotland Cancer Centre would like to thank Donna Crawford, Research Nurse, for her management of drug administration, sample collection and transportation, and arrangement of required scans. Support for third-party writing assistance for this article, furnished by Fiona Williams and Jamie Ashman, was provided by Prism Ideas. The four clinical centres participating in this study all acknowledge support from Cancer Research UK/Department of Health (England)/Chief Scientist Office (Scotland) as Experimental Cancer Medicine Centres, NIHR Biomedical Research Centres and for UCLH, the NIHR UCL/UCLH Clinical Research Facility.

\section{AUTHOR CONTRIBUTIONS}

Conception and design: R.K., R.P., A.H., J.L., L.R.M., A.S.-H., T.K., H.A.L., J.E., S.A., M.E. Development of methodology: R.K., F.B., A.T., M.R., N.T., M.E. Acquisition of data: R.K., S.S., N.F.B., R.R., F.B., A.T., R.P., U.A., J.L., M.R., N.T., J.E., L.R.M., H.S., N.R.M.H., M.F., N.D., A.G. Analysis and interpretation of data: R.K., A.H., N.F.B., F.B., P.J.L., A.T., R.P., J.L., A.S.H., M.R., N.T., H.S., N.R.M.H., M.F., N.D., A.G., T.K., H.A.L., J.E., L.R.M., S.A., M.E. Writing, review and/or revision of the manuscript: R.K., A.H., S.H., N.F.B., R.R., P.J.L., A.T., R.P., U.A., J.L., M.R., N.T., H.S., N.R.M.H., M.F., N.D., A.G., T.K., H.A.L., F.B., J.E., L.R.M., S.A., M.E. Study supervision: R.K., R.P., J.L., L.R.M., J.E., M.E.

\section{ADDITIONAL INFORMATION}

Ethics approval and consent to participate Prior to the study, ethics committee and institutional review board approval were obtained from the East of England (Essex) Research Ethics Service (Approval number 11/EE/0092). All patients provided written informed consent prior to study participation. The study was conducted in accordance with the Declaration of Helsinki and Good Clinical Practice.

Data availability The study data for this trial are considered commercially proprietary and are not available for unrestricted access. All the authors had full access to all the data in the study and take responsibility for the integrity of the data and the accuracy of the data analysis.

Competing interests R.K. holds a consulting agreement with Basilea Pharmaceutica International Ltd, outside of the submitted work that is not related to this compound. All Principal Investigators (R.K., J.L., R.P., J.E.) report receiving institutional reimbursement of clinical trial costs from Basilea Pharmaceutica International Ltd during the conduct of the study. J.E. is co-editor of the Clinical Subjects section of the British Journal of Cancer. A.G. is an Editorial Board Member of the British Journal of Cancer. S.A., F.B., T.K., H.L., P.J.L., A.S.-H., and M.E. are past or present employees of Basilea Pharmaceutica International Ltd and hold stock options. A.H. reports personal fees from Basilea Pharmaceutica International Ltd. during the conduct of the study. No potential conflicts of interest were disclosed by the other authors.

Funding information This study and editorial assistance for the preparation of this manuscript were supported by Basilea Pharmaceutica International Ltd. The study teams at the participating sites received infrastructure support from the ECMC (Experimental Cancer Medicine Centre) network, funded by Cancer Research UK, National Institute for Health Research including the NIHR/Wellcome UCL Clinical Research Facility, UCH/UCL Biomedical Research Centre and the Chief Scientist Office (Scotland).

Supplementary information is available for this paper at https://doi.org/10.1038/ s41416-020-1010-8.

Publisher's note Springer Nature remains neutral with regard to jurisdictional claims in published maps and institutional affiliations.

\section{REFERENCES}

1. Loong, H. H. \& Yeo, W. Microtubule-targeting agents in oncology and therapeutic potential in hepatocellular carcinoma. Onco. Targets Ther. 7, 575-585 (2014).

2. Dumontet, C. \& Jordan, M. A. Microtubule-binding agents: a dynamic field of cancer therapeutics. Nat. Rev. Drug Discov. 9, 790-803 (2010).

3. Mukhtar, E., Adhami, V. M. \& Mukhtar, H. Targeting microtubules by natural agents for cancer therapy. Mol. Cancer Ther. 13, 275-284 (2014).

4. Sawaguchi, Y., Ueno, S., Nishiyma, Y., Yamazaki, R. \& Matsuzaki, T. Establishment of a novel in vitro model for predicting incidence and severity of microtubuletargeting agent-induced peripheral neuropathy. Anticancer Res. 35, 6431-6437 (2015).

5. McGrogan, B. T., Gilmartin, B., Carney, D. N. \& McCann, A. Taxanes, microtubules and chemoresistant breast cancer. Biochim. Biophys. Acta 1785, 96-132 (2008).

6. Estève, M. A., Carré, M., Bourgarel-Rey, V., Kruczynski, A., Raspaglio, G., Ferlini, C. et al. Bcl-2 down-regulation and tubulin subtype composition are involved in resistance of ovarian cancer cells to vinflunine. Mol. Cancer Ther. 5, 2824-2833 (2006).

7. McCarroll, J. A., Gan, P. P., Liu, M. \& Kavallaris, M. betalll-tubulin is a multifunctional protein involved in drug sensitivity and tumorigenesis in non-small cell lung cancer. Cancer Res. 70, 4995-5003 (2010).

8. Pohlmann, J., Bachmann, F., Schmitt-Hoffmann, A., Gebhardt, K., Spickermann, J., Nuoffer, C., Biringer, G. Ã., Reilly, T., Pruschy, M. \& Lane, H. A. BAL101553: an optimized prodrug of the microtubule destabilizer BAL27862 with superior antitumor activity. Cancer Res. 71, abstract 1347 (2011).

9. Prota, A. E., Danel, F., Bachmann, F., Bargsten, K., Buey, R. M., Pohlmann, J. et al. The novel microtubule-destabilizing drug BAL27862 binds to the colchicine site of tubulin with distinct effects on microtubule organization. J. Mol. Biol. 426, 1848-1860 (2014).

10. Bachmann, F., Burger, K. \& Lane, H. BAL101553 (prodrug of BAL27862): the spindle assembly checkpoint is required for anticancer activity. Cancer Res. 75, abstract 3789 (2015).

11. Bergès, R., Tchoghandjian, A., Honore, S., Esteve, M. A., Figarella-Branger, D., Bachmann, F. et al. The novel tubulin-binding checkpoint activator BAL101553 inhibits EB1-dependent migration and invasion and promotes differentiation of glioblastoma stem-like cells. Mol. Cancer Ther. 15, 2740-2749 (2016).

12. Kolb, E. A., Gorlick, R., Keir, S. T., Maris, J. M., Kang, M. H., Reynolds, C. P. et al. Initial testing (stage 1) of BAL101553, a novel tubulin binding agent, by the pediatric preclinical testing program. Pediatr. Blood Cancer 62, 1106-1109 (2015). 
Phase 1/2a trial of intravenous BAL101553, a novel controller of the... R Kristeleit et al.

13. Mladek, A. C., Pokorny, J. L., Lane, H., Bachmann, F., Schroeder, M. A., Bakken, K. K., Carlson, B. L., Decker, P. A., Eckel-Passow, J. E. \& Sarkaria, J. N. The novel tubulinbinding 'tumor checkpoint controller' BAL101553 has anti-cancer activity alone and in combination treatments across a panel of GBM patient-derived xenografts. Cancer Res. 76, abstract 4781 (2016).

14. Sharma, A., Broggini-Tenzer, A., Vuong, V., Messikommer, A., Nytko, K. J., Guckenberger, $M$. et al. The novel microtubule targeting agent BAL101553 in combination with radiotherapy in treatment-refractory tumor models. Radiother. Oncol. 124, 433-438 (2017).

15. Bachmann, F., Burger, K., Duran, G. E., Sikic, B. I. \& Lane, H. A. BAL101553 (prodrug of BAL27862): a unique microtubule destabilizer active against drug refractory breast cancers alone and in combination with trastuzumab. Cancer Res. 74, abstract 831 (2014).

16. Bergès, R., Tchoghandjian, A., Sergé, A., Figarella-Branger, D., Bachmann, F. \& Lane, H. EB1-dependent long survival of glioblastoma cancer stem-like cell tumor-bearing mice after daily oral treatment with the novel tumor checkpoint controller BAL101553. Eur. J. Can. 103, abstract A166 (2018).

17. Forster-Gros, N., Bachmann, F., McSheey, P. \& Lane, H. A. BAL101553, a novel microtubule-targeting tumor checkpoint controller, in combination with eribulin leads to increased cures in a TNBC xenograft model. Eur. J. Can. 103, abstract LBA11 (2018).

18. Lane, H., McSheehy, P. \& Bachmann, F. BAL101553, a novel microtubule-targeting tumor checkpoint controller, synergizes with gemcitabine providing cures in a PDX-pancreatic model. Eur. J. Can. 103, abstract LBA16 (2018).

19. Sharma, A., Bachmann, F., Broggini-Tenzer, A., Guckenberger, M., Lane, H. \& Pruschy, M. N. The novel tubulin-binding, tumor checkpoint controller BAL101553 has differential effects on tumor vascularization with IV and oral dosing and provides superior anti-tumor activity in combination with bevacizumab. Cancer Res. 77, abstract LB151 (2017).
20. Duran, G. E., Lane, H., Bachmann, F. \& Sikic, B. I. In vitro activity of the nove tubulin active agent BAL27862 in MDR1(+) and MDR1(-) human breast and ovarian cancer variants selected for resistance to taxanes. Cancer Res. 70, abstract 4412 (2010).

21. Bachmann, F. \& Lane, H. Dual mechanism of action of the novel microtubuletargeting drug BAL27862 (active moiety of the prodrug BAL101553): targeting tumor and vascular cells. Eur. J. Can. 48, abstract 421 (2012).

22. Carlson, K. \& Ocean, A. J. Peripheral neuropathy with microtubule-targeting agents: occurrence and management approach. Clin. Breast Cancer 11, 73-81 (2011).

23. Joerger, M., Stathis, A., Metaxas, Y., Hess, D., Mantiero, M., Mark, M. et al. A phase 1 study of BAL101553, a novel tumor checkpoint controller targeting microtubules, administered as 48-h infusion in adult patients with advanced solid tumors. Invest. New Drugs. 38, 1067-1076 (2020).

Open Access This article is licensed under a Creative Commons Attribution 4.0 International License, which permits use, sharing, adaptation, distribution and reproduction in any medium or format, as long as you give appropriate credit to the original author(s) and the source, provide a link to the Creative Commons license, and indicate if changes were made. The images or other third party material in this article are included in the article's Creative Commons license, unless indicated otherwise in a credit line to the material. If material is not included in the article's Creative Commons license and your intended use is not permitted by statutory regulation or exceeds the permitted use, you will need to obtain permission directly from the copyright holder. To view a copy of this license, visit http://creativecommons. org/licenses/by/4.0/.

(c) The Author(s) 2020 